\title{
Bone mineralisation in preterm infants measured by dual energy radiographic densitometry
}

\author{
A J LYON, ${ }^{*}$ D J HAWKES, $\uparrow \mathrm{M}$ DORAN,$\dagger \mathrm{N}$ McINTOSH, ${ }^{*}$ AND F CHAN* \\ ${ }^{*}$ Neonatal Unit and †Department of Medical Physics and Bioengineering, St George's Hospital, London
}

SUMmaRY Dual energy radiographic densitometry was used to follow postnatal changes in the bone mineral content of the radii of 15 infants of less than 30 weeks' gestation. The system permitted bone mineral content to be measured with minimal disturbance to the infants in their incubators. Mean bone mineral content at birth was $2.4 \mathrm{mg} / \mathrm{mm}$ shaft length, decreasing to $1.9 \mathrm{mg} / \mathrm{mm}$ at 6 weeks of age, before starting to rise. Mineralisation was poor compared with that of a fetus at an equivalent postconceptual age. Mean intakes of calcium and phosphate were considerably less than the intrauterine accumulation of these minerals and it is postulated that this was the main cause of the poor mineralisation. Radiographic densitometry is both accurate and precise and has advantages over photon absorptiometry in that it can be used to measure bone mineral in infants who are not only preterm, but also ill enough to require intensive care.

The bones of a preterm infant are shorter, lighter, and contain less calcium/unit length than those of a fetus at an equivalent gestational age. ${ }^{1}$ Osteopenia and rachitic changes are frequent radiographic findings, and spontaneous fractures are common. Biochemical abnormalities, particularly hypercalcaemia, hypophosphataemia, and increased alkaline phosphatase activity, have been reported in association with problems of bone mineralisation in premature babies. ${ }^{2}$

Identifiable radiographic changes are late manifestations of the metabolic bone disease of prematurity and are non-specific. To study this problem further a method for measuring bone mineral content is needed. Photon absorptiometry is both sensitive and precise and has been used by several workers. ${ }^{34}$ The equipment is, however, expensive and its bulk makes it impractical for measurements on babies who are too ill to be removed from their incubators.

We have developed a system for measuring bone mineral content in preterm infants using dual energy radiographic densitometry that can be used on preterm infants while they are undergoing intensive care. $^{5}$

The aim of this study was to use this technique to measure and follow the postnatal changes in bone mineral content of the radii of preterm infants.

\section{Patients and methods}

Infants of less than 30 weeks' gestation were studied. Informed consent was obtained from the parents.

Dual energy radiographs at $40 \mathrm{kv}$ and $120 \mathrm{kv}$ were taken of the forearm within three days of birth, at 7 days of age, and then weekly until discharge from the unit. The arm was held in a plastic holder that incorporated a calibration wedge with steps of aluminium and perspex of varying thicknesses. The radiographs were taken with the mobile camera routinely used on the neonatal unit and with minimal disturbance to the infants in their incubators.

Records were kept of the intravenous and oral intakes of calcium and phosphate, but in this study no changes were made to the feeding policy of the unit. Weight was recorded weekly and the length of each radius measured directly from the radiographs. The films were digitised by computer. The effect of soft tissue was subtracted out by the use of low and high energy films. Comparison with the step wedge allowed the image of the radius to be converted into an equivalent mass of aluminium and perspex. The mineral content of the radius expressed as $\mathrm{mg}$ of calcium and phosphorus $/ \mathrm{mm}$ shaft length was then calculated using known values for the radiographic 
Table Details of 15 infants. Values are expressed as mean (range)

\begin{tabular}{lll}
\hline $\begin{array}{l}\text { Gestation } \\
\text { (weeks) }\end{array}$ & $\begin{array}{l}\text { Birth weight } \\
(\mathrm{g})\end{array}$ & $\begin{array}{l}\text { Period studied } \\
\text { (weeks) }\end{array}$ \\
\hline $27 \cdot 3(25-29)$ & $1133(820-1480)$ & $9(5-13)$ \\
\hline
\end{tabular}

mass attentuation coefficients of aluminium, perspex, and bone. ${ }^{5}$

The total mineral content of the distal half of each radius was measured and from this the average bone mineral content $/ \mathrm{mm}$ shaft length was obtained. Changes in the mineral content of the metaphysis of each bone were also measured at a point $2 \mathrm{~mm}$ from the distal end.

\section{Results}

Fifteen infants were studied (table), and bone mineral content varied along the shaft of the radius (fig 1). On all radiographs there was a dense band at the distal end of the bone and, for measurement purposes, this first peak (A) was taken as marking the start of the bone. Proximal to this peak there was an area of lower density (B), and this region showed the greatest changes in mineral content with postnatal age.

Mineral content measured at birth increased with increasing gestational age (fig 2). This figure also includes data from four infants of gestational ages of 30 weeks or more. The values lie close to the curve published by Greer et al using photon absorptiometry, ${ }^{6}$ which shows the mean bone mineral

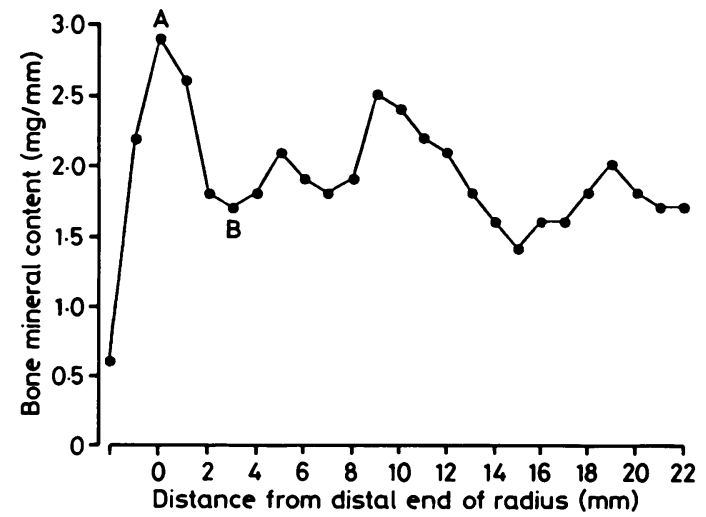

Fig 1 Plot of bone mineral content $(\mathrm{mg} / \mathrm{mm})$ along distal half of radius. $A=$ first peak; $B=$ area of lower density.

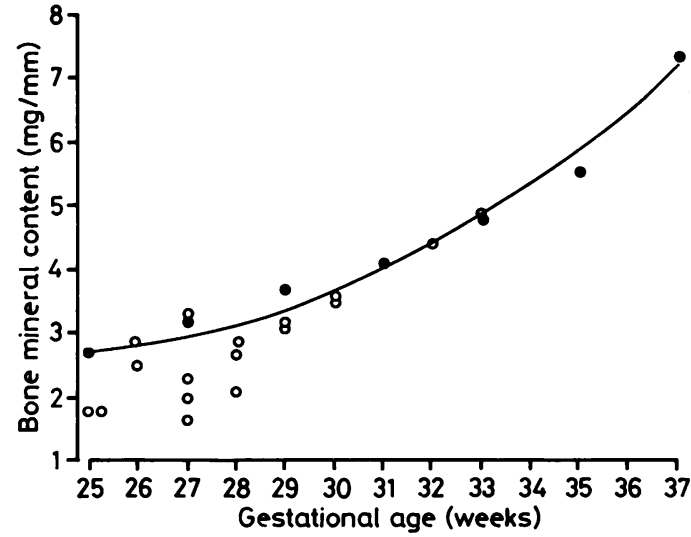

Fig 2 Bone mineral content of individual infants at birth plotted against gestational age (open circles) compared with data obtained by photon absorptiometry (closed circles). ${ }^{6}$

content of newborn infants at various gestational ages. At the lower gestational ages the measurements from this study are lower than those of Greer $e t a l$, but all the points lie within two standard deviations of the published mean values.

In figs 3 and 4 it can be seen that the bone mineral content of the metaphysis and the average mineral content of the shaft of the radius changed with postnatal age. In each figure the changes are compared with an 'intrauterine' standard. This has been calculated by taking an 'average' infant from the group studied (that is, 27 weeks as shown in the

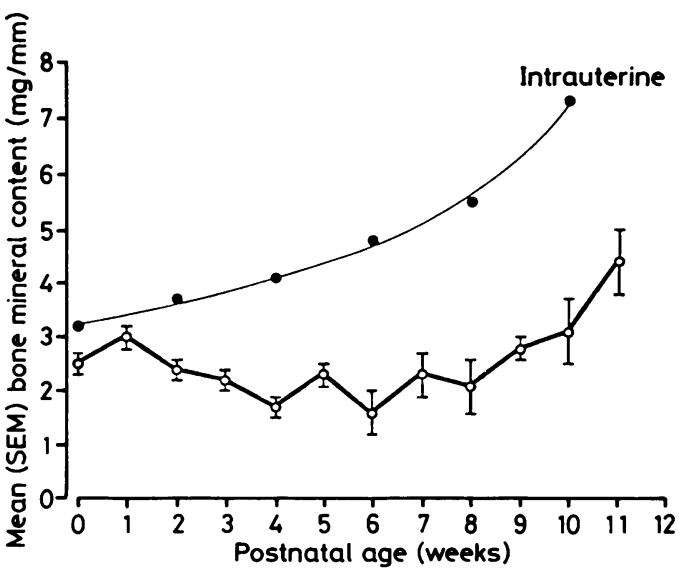

Fig 3 Changes in mean (SEM) bone mineral content of the metaphysis with postnatal age. Expected fetal mineralisation shown in upper trace. 


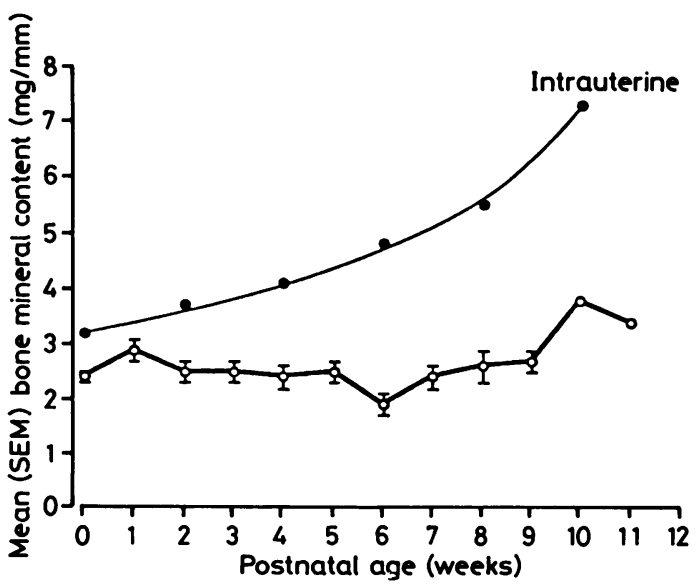

Fig 4 Changes in mean (SEM) bone mineral content of shaft of radius with postnatal age. Expected fetal mineralisation shown in upper trace.

table), and, using the data of Greer et al, plotting what would have happened to its bone mineralisation had it remained in the uterus. It can be seen that had the infants remained in utero, mineralisation would have been significantly greater than was achieved after birth.

The data for the first six weeks of life in figs 3 and 4 are similar. At birth the mean (SEM) bone mineral content was $2.4(0.1) \mathrm{mg} / \mathrm{mm}$, rising to 3.0 $(0 \cdot 2) \mathrm{mg} / \mathrm{mm}$ by 6 days of age. There was then a fall to a minimum of $1.9(0.2) \mathrm{mg} / \mathrm{mm}$ at 6 weeks, followed by a steady rise in the mineral content of the metaphysis (fig 3). The average bone mineral content of the shaft also increased after 6 weeks, but the rise was less pronounced than that seen in the metaphysis (fig 4).

Despite the apparent poor mineralisation the infants continued to gain weight and increase bone length (fig 5). Birth weight was regained by 10 to 14 days, followed by a mean weight gain of $14 \mathrm{mg} / \mathrm{kg} /$ day (similar to that of the fetus). From birth the radius increased in length by about $1.5 \mathrm{~mm} /$ week.

Using an 'average' infant from the group studied, the data in fig 6 show the expected accumulation of minerals had it remained in the uterus ${ }^{7}$ and this is compared with the actual intakes of calcium and phosphate plotted against postnatal age. Not all the

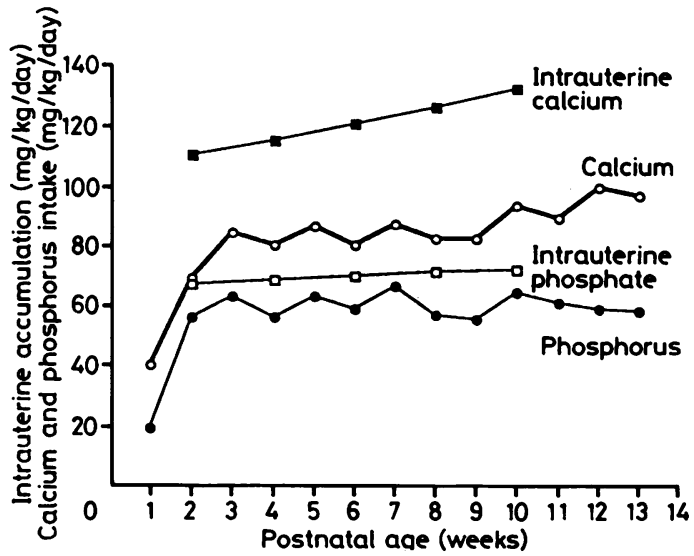

Fig 6 Mean intakes of calcium and phosphate plotted against postnatal age and compared with calculated intrauterine accumulations. $^{7}$

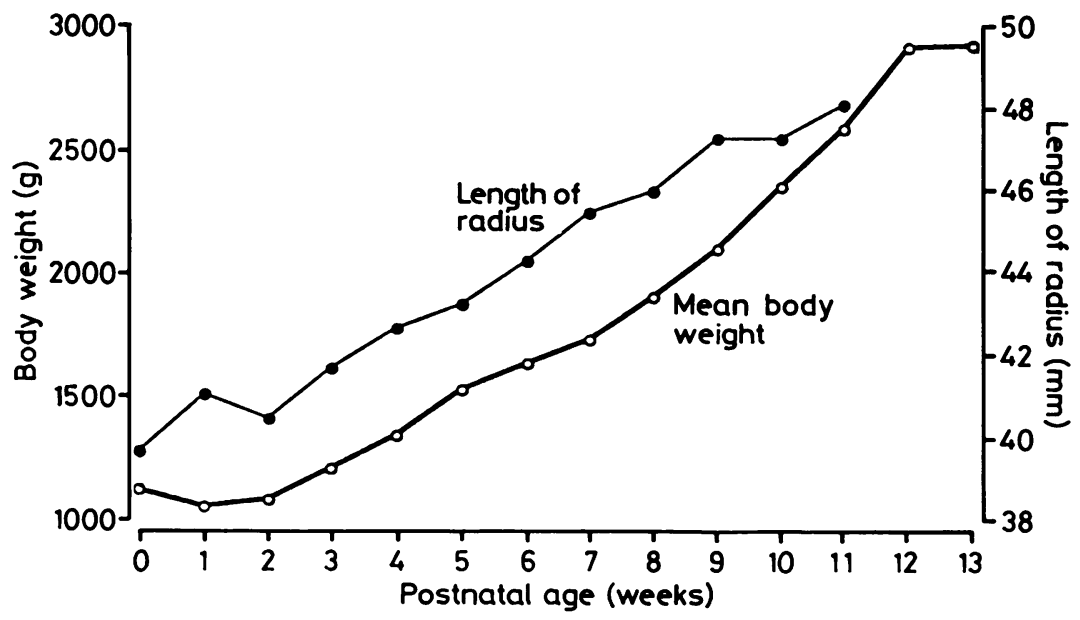

Fig 5 Change in mean body weight and length of radius with postnatal age. 
dietary mineral would be retained, so the actual accretion would be significantly less than the intakes shown in the figure and much lower than the amounts the infant would have received if it had still been in the uterus.

No changes were made to the feeding policy of the unit. Preterm infants were initially fed with expressed breast milk or parenteral nutrition if oral feeds were not tolerated. Once feeding was established, expressed breast milk or a preterm formula was used. Mineral supplements were added to the breast milk at the discretion of the medical staff. All infants were given 1000 units of vitamin $\mathrm{D}$ a day. There was no correlation between intake and mineral content of the radius.

\section{Discussion}

The use of dual energy radiographic densitometry has permitted the measurement of bone mineral in preterm infants, even when they are sick and undergoing intensive care. The film, holder, and calibration wedge fit easily into an incubator and the baby needs the minimum of handling. Radiation dosage is low, a whole body equivalent of $0.4 \mu \mathrm{Sv}$ compared with $17 \cdot 4 \mu \mathrm{Sv}$ for a chest radiograph.

Using photon absorptiometry, mineral content is measured in a short segment of bone. ${ }^{34}$ The data in fig 1 , however, show that mineral content is not uniform along the shaft of the bone. In longitudinal studies it is important to scan the same segment of bone on each occasion, and this may be difficult to locate using skin surface markings. Data in figs 3 and 4 show that changes in one part of the bone may not reflect what is happening in the bone as a whole. Changes in mineral content of the metaphysis occurred earlier and were more pronounced than in the rest of the bone. This is perhaps not surprising as the metaphysis is the most active part of the bone.

A radiograph easily relates measurement to a fixed position on the bone. It is also possible to measure mineral at various points on the bone, or to calculate the total bone mineral content. Any bone may be used as long as it can be immobilised to allow two radiographs to be taken with the bone in the same position. In this study the distal half of the radius was used but with minor alterations to the technique it would be possible to measure total mineral content in both forearm bones, which will reduce the effect of variations along a bone or between different bones.

The data in fig 2 show that there is reasonable correlation between bone mineral measurements obtained using radiographic densitometry and those from photon absorptiometry, and this supports the other validation studies of the system..$^{5}$ At the lower gestational ages, however, the results from this study appear to be consistently below those published by Greer $e t$ al. This may be due to differences in the racial composition of the two populations. In the study of Greer $e$ al the patients were predominantly white $(92 \%)$, but in this study eight were white and seven Asian. At less than 35 weeks' gestation Asian infants have lower bone mineral contents than white babies, ${ }^{8}$ and this may have influenced the results in this study.

During the first week of life there was a rise in bone mineral content in every baby studied. During the first few days of life preterm infants showed a fall in plasma calcium concentration, possibly due to low parathyroid hormone and to high circulating concentrations of calcitonin. ${ }^{9}$ The initial rise in mineral content could well be a result of these hormonal changes. Over the next 4 to 6 weeks of life the bone mineral content decreased. The bones continued to increase in length, suggesting that matrix was being laid down even if bone mineralisation was poor.

The data in fig 5 show that these babies had mineral intakes considerably lower than a fetus of equivalent postconceptual age, and this was probably the main reason for the poor mineralisation. After 6 weeks bone mineral content increased even though there was no change in dietary intake of calcium and phosphate. This may have been due to an improvement in the general health of the baby or to increased retention of mineral as a result of better gut absorption and lower renal losses. ${ }^{10} 11$

The fact that bone mineral content improved spontaneously does not mean that a period of suboptimal mineralisation is acceptable. The long term consequences are not known. Dental defects have been associated with neonatal hypocalcaemia and it has been postulated that transient disturbances in calcium metabolism at a sensitive period of tooth formation can have serious effects on enamal calcification. ${ }^{12}$ In the short term, better mineralisation should reduce the chances of spontaneous fractures and lessen the possibility of rachitic respiratory distress. ${ }^{13}$

Nutrition groups in America ${ }^{14}$ and Europe ${ }^{15}$ now recommend intakes of calcium of $180-210 \mathrm{mg} / \mathrm{kg} / \mathrm{day}$ and phosphate of $120-140 \mathrm{mg} / \mathrm{kg} / \mathrm{day}$, which are far in excess of the intakes given in this study and greater than those given in most neonatal units in Britain. There is some evidence that in preterm infants who are otherwise well these higher intakes result in better bone mineralisation. ${ }^{16}$

There may, however, be a problem in improving mineral retention in sick preterm babies who need intensive care. These infants are often parenterally fed for long periods and it is difficult to keep high 
concentrations of calcium and phosphate in solution. Problems such as patent ductus arteriosus are often managed by fluid restriction, with subsequent reduction in the intakes of all nutrients including calcium and phosphorus.

Dual energy radiographic densitometry is a convenient method for accurately measuring and following changes in the bone mineral content of preterm infants. It entails minimal handling and will be used in further studies to measure the effect of diet on the mineralisation of those infants who are not only preterm but also ill enough to require intensive care.

We are grateful to Dr J Williams and the radiographers for all the radiography and to Mrs B Gagen for typing the manuscript. F Chan was supported by a grant from Milupa.

\section{References}

${ }^{1}$ McIntosh N, Shaw JCL, Taghizadeh A. Direct evidence for calcium and trace mineral deficits in the skeleton of preterm infants. Pediatr Res 1974;8:896.

${ }^{2}$ Lyon AJ, McIntosh N, Wheeler K, Brooke OG. Hypercalcaemia in extremely low birthweight infants. Arch Dis Child 1984;59: $1141-50$.

3 James JR, Truscott J, Congdon PJ, Horsman A. Measurement of bone mineral content in the human fetus by photon absorptiometry. Early Hum Dev 1986;13:169-81.

${ }^{4}$ Greer FR, Lane J, Weiner S, Mazess RB. An accurate and reproducible absorptiometric technique for determining bone mineral content in newborn infants. Pediatr Res 1983;17:259-62.

5 Hawkes DJ, Lyon AJ, Doran M, McIntosh N. A technique to measure the bone mineralisation of preterm infants using digitised dual energy radiographs. Med Phys (in press).

6 Greer FR, McCormack A. Bone growth with low mineral content in very low birthweight premature infants. Pediatr Res 1986;20:925-8.

7 Shaw JCL. Malnutrition in very low birthweight, preterm infants. Proc Nutr Soc 1974;33:103-11.

8 Ryan S, Congdon PJ, James J, Truscott J, Horsman A. Mineral accretion in the human fetus. Arch Dis Child 1988;63:799-808.

9 Romagnoli C, Zecca E, Tortorolo G, Diodata A, Fazzini G, Sorcini-Carta M. Plasma thyrocalcitonin and parathyroid hormone concentrations in early neonatal hypocalcaemia. Arch Dis Child 1987;62:580-4.

${ }^{10}$ Shaw JCL. Evidence for defective skeletal mineralisation in low birthweight infants: the absorption of calcium and fat. Pediatrics 1976;57:16-25.

1 Lyon AJ, McIntosh N. Calcium and phosphorus balance in extremely low birthweight infants in the first six weeks of life. Arch Dis Child 1984;59:1145-50.

12 Stimmler L, Snodgrass GJAI, Jaffe E. Dental defects associated with neonatal symptomatic hypocalcaemia. Arch Dis Child 1973;48:217-20.

13 Glasgow JFT, Thomas PS. Rachitic respiratory distress in small premature infants. Arch Dis Child 1977;52:268-74.

14 American Academy of Pediatrics: Committee on Nutrition. Nutritional needs of low-birthweight infants. Pediatrics 1985;75: 976-86.

15 ESPGAN Committee on Nutrition. Nutrition and feeding of preterm infants. Acta Paediatr Scand 1987;336(suppl):9-10.

16 Chan GM, Mileur L, Hansen JW. Effects of increased calcium and phosphorus formulas and human milk on bone mineralisation in preterm infants. J Pediatr Gastroenterol Nutr 1986;5:444-9.

Correspondence and requests for reprints to $\mathrm{Dr} \mathrm{AJ}$ Lyon, Paediatric Department, Mayday University Hospital, Mayday Road, Thornton Heath, Surrey CR4 7YE.

Accepted 24 January 1989 